
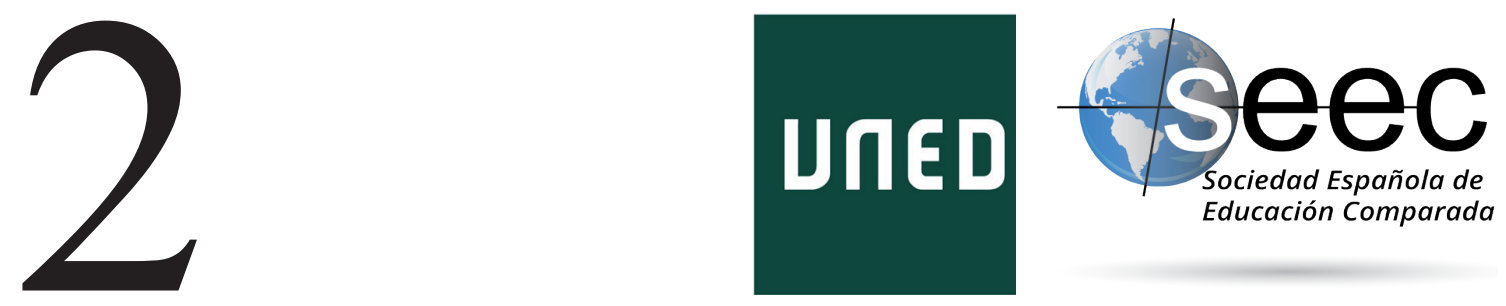

\title{
Notas para una construcción metodológica en educación comparada
}

\author{
Notes for a metodological construction \\ in comparative education
}

\section{Patricia Ducoing-Watty*; Ileana Rojas-Moreno**}

DoI: $10.5944 /$ reec.30.2017.17766

\section{Recibido: 21 de diciembre de 2016 Aceptado: 16 de agosto de 2017}

\footnotetext{
* Patricia Ducoing-Watty: Doctora en Pedagogía por la Universidad Nacional Autónoma de México. Investigadora titular «C» tiempo completo y del posgrado en Pedagogía de la Facultad de Filosofía y Letras de la UNAM. Investigadora nacional Nivel 2 del Sistema Nacional de Investigadores-CONACYT. Líneas de investigación: formación docente y formación universitaria en educación, educación secundaria y educación comparada. Autora de publicaciones nacionales e internacionales sobre educación e investigación educativa. Docente y coordinadora en la impartición de cursos sobre formación y actualización de docentes de los niveles básico, medio, superior y posgrado en instituciones nacionales e internacionales. Datos de contacto: E-mail: pducoingw@gmail.com

* Ileana Rojas-Moreno: Doctora en Ciencias con especialidad en Investigaciones Educativas del Departamento de Investigaciones Educativas del CINVESTAV-IPN. Profesora-investigadora de carrera titular «A» tiempo completo y del posgrado en Pedagogía de la Facultad de Filosofía y Letras de la UNAM. Investigadora nacional Nivel 1 del Sistema Nacional de Investigadores-CONACYT. Líneas de investigación: formación universitaria en educación, educación comparada y construcción de campos disciplinarios. Autora de publicaciones nacionales e internacionales sobre educación e investigación educativa. Docente y coordinadora en la impartición de cursos sobre formación y actualización de docentes de los niveles básico, medio, superior y posgrado en instituciones nacionales e internacionales. Datos de contacto: E-mail: ileana_rojas_moreno@hotmail.com
} 


\title{
Resumen
}

Este artículo se elaboró en el marco del Programa de Apoyo a Proyectos de Investigación e Innovación Tecnológica de la Universidad Nacional Autónoma de México (Proyecto PAPIIT IN-402712). Su abordaje alude a varios aspectos de carácter teórico-metodológico con la finalidad de puntualizar algunas reflexiones en torno a la construcción de estrategias de investigación comparada. Para tal efecto, presentamos como referente su utilización en un objeto de investigación específico: la educación secundaria en Europa y América Latina (casos de Francia y Brasil). Su delimitación está basada en una selección justificada de países, a partir de la cual argumentamos que este ciclo educativo se configura como un objeto único y singular en el marco de la diversidad; al mismo tiempo, dicho ciclo se muestra como un espacio formativo común al encontrarse institucionalizado internacionalmente en tanto un nivel de estudios de la educación básica. En el contenido de este documento incluimos como puntos nodales los siguientes: a) breve desarrollo de notas para una construcción metodológica de corte comparativo; b) caracterización de un diseño de investigación para derivar estrategias de contraste; y c) presentación de algunos de los resultados más representativos de una investigación acerca de la educación secundaria en Francia y Brasil, basada en la aplicación de la propuesta metodológica definida. A manera de cierre incluimos una breve discusión sobre el proceso de construcción de una metodología ad hoc.

Palabras clave: educación comparada; metodología comparativa en educación; educación secundaria; sistemas educativos; política educativa

\begin{abstract}
This article was elaborated within the framework of the Support Program for Research and Technological Innovation Projects of the National Autonomous University of Mexico (Project PAPIIT IN-402712). His approach refers to several theoretical and methodological aspects in order to point out some reflections regarding building strategies and comparative research. For this purpose, we present as a reference the use in a specific research object: secondary education in Europe and Latin America (cases of France and Brazil). Its delimitation is based on a justified selection of countries, from which it we argue that this educational cycle is configured as a unique and singular object within the framework of diversity; at the same time, this cycle is shown as a common formative space to find itself institutionalized internationally as a level of studies of basic education. In the contents of this document we include as nodal points the following: a) brief development of notes for a methodological construction of comparative cut; b) characterization of a research design to derive contrast strategies; and c) presentation of some of the most representative results of research about secondary education in France and Brazil, based on the application of the defined methodological proposal. In closing, we include a brief discussion of the process of building an ad hoc methodology.
\end{abstract}

Key Words: comparative education; comparative method in education; secondary education; education systems; educational policy 


\section{Introducción}

Diversos autores destacan que durante el transcurso de poco más de dos siglos la investigación comparada gradualmente ha adquirido un mayor auge a partir de trabajos con tratamientos diversos tales como definición de sistemas modélicos, estudios histórico-culturales, programas de desarrollo social, definición de agendas de política internacional, entre otros (Cf. Altbach \& Kelly, 1990; Schriewer, 2011; Valle et al, 2016). Actualmente, en la investigación comparada se visualiza el mundo en su totalidad como una unidad de análisis aunque, por otra parte, se cuestionan fuertemente las suposiciones teóricas, epistémicas y metodológicas fundamentales de esta perspectiva como tal (Wallerstein, 2013). ${ }^{1}$ Acordes con estas reflexiones, los expertos en el campo coinciden en definir exigencias básicas para la construcción de estrategias metodológicas en la investigación comparada.

En lo que concierne a la educación comparada, partimos de situarla como un espacio para la investigación en el campo de conocimiento educativo y en el ámbito de las Humanidades y las Ciencias Sociales. Ya sea vista como rama, ámbito, programa o campo del conocimiento, a partir de la segunda mitad del siglo veinte la educación comparada ha tenido importantes avances en cuanto al desarrollo de enfoques teóricos y propuestas metodológicas. En el campo educativo, el impacto de las investigaciones de corte comparativo ha demostrado cada vez más una mayor presencia, particularmente al considerar el marco configurado por los procesos de internacionalización y globalización económica (Valle et al., 2016). De entre las diversas aportaciones de la educación comparada, el abordaje contrastante de sistemas y/o modelos educativos distintos ha respondido principalmente al propósito de caracterizar analíticamente las semejanzas y diferencias entre instituciones, entidades, países y regiones (Chnane-David y Groux, 2009; Schriewer, 1993, 2011, Valle et al., 2016).

En relación con estos señalamientos y mediante la vinculación con los avances de una investigación institucional en proceso, abordamos a continuación aspectos de índole metodológica a fin de derivar algunas anotaciones sobre la construcción de estrategias teórico-metodológicas para la educación comparada. Para efectos del presente artículo nos interesa mostrar su aplicación en una investigación de corte comparativo sobre la educación secundaria en Europa y América Latina, mediante la elección de dos casos, Francia y Brasil, seleccionados de un universo de estudio más amplio por tratarse de países representativos de contextos regionales contrastantes (Unión Europea y Región Latinoamericana, respectivamente) y con un desarrollo diferenciado de sus respectivos sistemas educativos. ${ }^{2}$ Desde nuestra perspectiva, argumentamos que este

$1 \quad$ Desde finales del siglo XVIII la utilización de la metodología comparada representó la vanguardia y el impulso en la constitución y demarcación de campos disciplinarios como el derecho, la medicina, la lingüística, la educación, funcionando como un elemento esencial en los procesos de reestructuración de largo alcance de la ciencia moderna. Es hacia finales del siglo XX y principios del nuevo milenio que podemos observar cómo, de una visión de mundo concebido como una diversidad de sociedades regionales o nacionales separadas, se ha transitado a la mirada de entidades autónomas, de configuraciones históricamente caracterizadas, de ambientes dependientes unos de otros.

$2 \quad$ Hacemos mención del Proyecto de Investigación Institucional PAPIIT IN 402712, realizado con el apoyo de la Universidad Nacional Autónoma de México. En extenso, los países que conformaron la muestra total fueron los siguientes: De Europa, los casos de Alemania, Austria, Bélgica, España, Francia e Inglaterra. De América Latina, los casos de Argentina, Bolivia, Brasil, Chile, Colombia, Costa Rica, Guatemala, México, Perú, Uruguay y Venezuela. 
ciclo educativo es susceptible de configurarse como un objeto único y singular en el marco de la diversidad, a la vez que se presenta como un espacio formativo común al encontrarse institucionalizado internacionalmente en términos de un nivel de estudios de la educación básica.

Este documento incluye tres apartados. En el primero desarrollamos breves anotaciones metodológicas de corte comparativo. En el segundo apartado proponemos un diseño sobre una estructura de contraste y la derivación de estrategias de búsqueda específicas. Basándonos en la aplicación del citado diseño, en el tercer apartado mostramos algunos de los resultados más representativos de la investigación de referencia para caracterizar la educación secundaria en los casos de Francia y Brasil. Por último, proponemos algunos puntos para la discusión sobre la construcción metodológica en educación comparada.

\section{Notas sobre metodología comparada}

De entre la diversidad de textos y documentos de análisis respecto de la construcción metodológica en investigación comparada, encontramos cierta variedad tanto de reflexiones teóricas como de argumentaciones sobre estrategias de esta índole, tomando incluso como base los resultados de investigaciones nacionales e internacionales. De esta diversidad de insumos, retomamos algunos planteamientos específicos de Ball (citado por Beech y Meo, 2016), Steiner-Khamsi (2015), Schriewer (1993), Stake (2001) y Kin (1994) a fin de definir un esquema ordenador.

En lo que concierne a las intuiciones teóricas de Ball (2016), una investigación comparada -como por ejemplo sobre políticas educativas - tendría que abordar dos planos de análisis. En el primer plano habría de considerar, además de la demarcación de los Estados nacionales, la participación de nuevos actores tales como organismos supranacionales, empresas, Organizaciones No Gubernamentales (ONG), entre otros. Asimismo, en este plano se develarían aquellas implicaciones que influyen en la redefinición del papel del Estado y del establecimiento de nuevas formas de gobernar en educación. En el segundo plano tendrían que analizarse los ciclos de diseño e implantación de estrategias (p. ej., políticas educativas, modelos, programas) en los niveles macro y microinstitucional, procurando contrastar las visiones racionalistas y lineales en las que usualmente se sitúan la toma de decisiones de la macroplaneación y la puesta en marcha en la práctica educativa cotidiana.

En cuanto a los desarrollos de Stainer-Khamsi (2015) concretamente ubicados en el marco de la investigación comparada, retomamos el manejo de los conceptos de «recepción» y «traducción» para poder comprender la lógica de los sistemas educativos. Esto último mediante el análisis de los procesos de transferencia de políticas educativas. De acuerdo con la autora, una aproximación comparativa implica, por un lado, analizar detalladamente las diferencias fundamentales entre sistemas educativos y, por otro lado, investigar las formas a partir de las cuales los diversos actores (funcionarios, directivos, docentes, comunidades escolares) enfrentan los procesos de importación de agendas educativas internacionales (p. ej., estándares de evaluación y docencia, modelos académicos, reformas educativas, estrategias/competencias para el aprendizaje y la enseñanza, etc.), en particular de aquellas que han mostrado resultados contraproducentes agravando incluso problemas nacionales y/o locales pre-existentes. 
Respecto de las aportaciones de Schriewer (1993), retomamos las consideraciones de orden conceptual y metodológico que enfatizan el potencial de las aproximaciones comparatistas basadas en la categorización de «redes de interrelación» y en el manejo de la comparatividad multinivel. En el caso de la citada categorización, se trata de una idea-guía para aplicar la comparatividad que rebasa el ejercicio de relacionar fenómenos culturales entre sí (contenido fáctico), intentando descubrir «conexiones supuestamente hipotéticas», o bien «redes» entre distintos fenómenos, manifestaciones empíricas, variables identificables, niveles o sistemas, todos ellos concretados en forma de grupos de datos situados en varios contextos socioculturales. Posteriormente, de esos grupos de datos (unidades de referencia y/o comparación) se procede a «relacionar esas relaciones», contrastándolas con hipótesis para así ponderar su potencial de generalización teórica y sus alcances explicativos.

Por lo que toca a los aportes de Stake (2001) y Kin (1994), retomamos algunos planteamientos a modo de resaltar el basamento de los estudios de caso múltiple y su relación con los insumos previos. En este sentido reiteramos que la posibilidad de realización de los procedimientos de comparatividad entre identidades semejantes y diferentes está soportada en la utilización de los estudios de caso múltiples, en donde cada caso representa una unidad de análisis situada (de referencia y/o de comparación). Está demostrado que este tipo de abordaje permite comprender los vínculos de un proceso, de un fenómeno, de un objeto de investigación, en general, en su contexto. De acuerdo con Stake (2001), el estudio de caso posibilita el estudio de lo particular y lo complejo de un caso singular, para adentrarse en él y poder comprender tanto su actividad en circunstancias importantes como su articulación en otros contextos, es decir, su interrelación con ellos. Por su parte, Kin (1994) apunta que el estudio de casos consiste en una investigación empírica abocada al estudio de un fenómeno contemporáneo al interior de su contexto, en particular cuando los límites entre dicho fenómeno y el contexto de referencia no resultan tan evidentes. En breve, mediante los estudios de caso múltiples se tienen más probabilidades de aproximarse a los vínculos que unen los diversos elementos («redes de interrelación») que de sobreestimar el tema de las frecuencias estadísticas, posibilitando con ello el descubrimiento de tópicos recurrentes en el marco de un cierto número de diversos contextos históricos, culturales, sociales, políticos y económicos.

\section{Diseño y aplicación de una estructura metodológica de contraste}

Los planteamientos expuestos en el apartado anterior nos llevan a considerar su cualidad heurística en términos de encuadres para diseñar dispositivos de indagación, más que pretender una exposición detallada de modelos prescriptivos de una presumible ortodoxia comparatista. Desde esta mirada proponemos aquí, en primer lugar, una representación sucinta de posibles articulaciones entre los niveles de análisis (Gráfico 1). De acuerdo con esta esquematización, contamos con una vista panorámica de los diferentes niveles de comparatividad desde los cuales, y mediante la utilización de categorías analíticas vinculantes, situamos los planos de la investigación y los entrelazamientos posibles. Observamos también que en cada nivel se realizan tareas de investigación comunes de recopilación, sistematización y presentación descriptiva de información documental (gabinete), empírica (estadística) y/o de campo. 


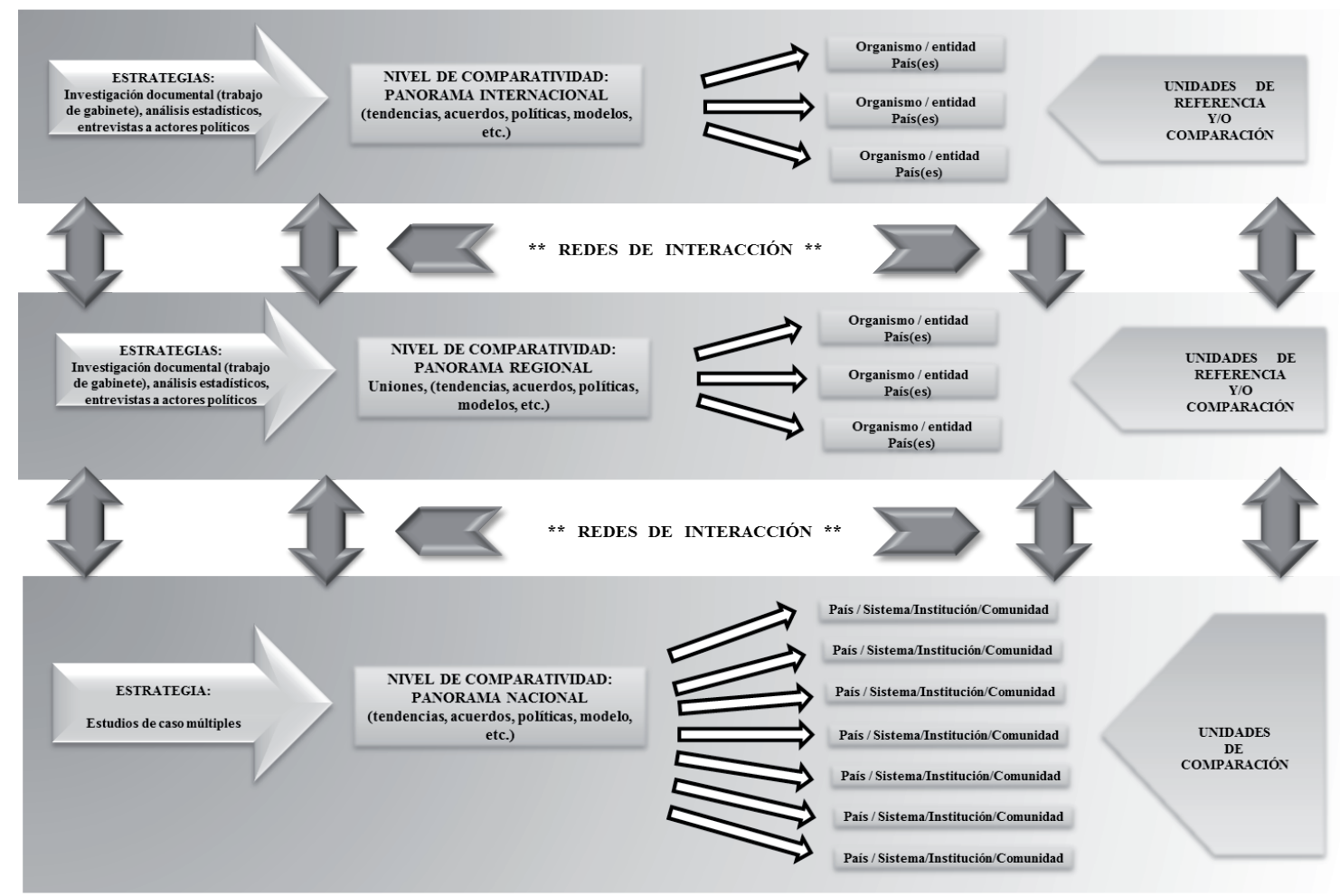

Gráfico 1: Niveles y unidades de comparatividad. Fuente. Elaboración propia.

En segundo lugar nos ocupamos del nivel nacional, y aquí el trabajo de soporte está representado por los estudios de caso múltiples, si bien esto último no significa que dicha estrategia sea privativa de este nivel. Más aún, dado que en cada nivel de estudio se delimitan grados de generalidad, profundidad y especificidad, una vía para obtener un entretejido de comprensión se deja entrever mediante el cuidado de dos rubros de la investigación: a) la contextualización sociohistórica (internacional, regional y nacional); y, b) la articulación multinivel mediante el manejo de categorías analíticas seleccionadas ex profeso. Para los estudios de caso de la investigación institucional de referencia, los dos incisos anteriores orientaron el desagregado de contenido que presentamos a continuación en el Esquema 1.

Esquema 1: Listado de aspectos básicos para los Estudios de Caso.

\section{x Marco sociohistórico}

o Perfil del país en la actualidad

o Economía

o Población 


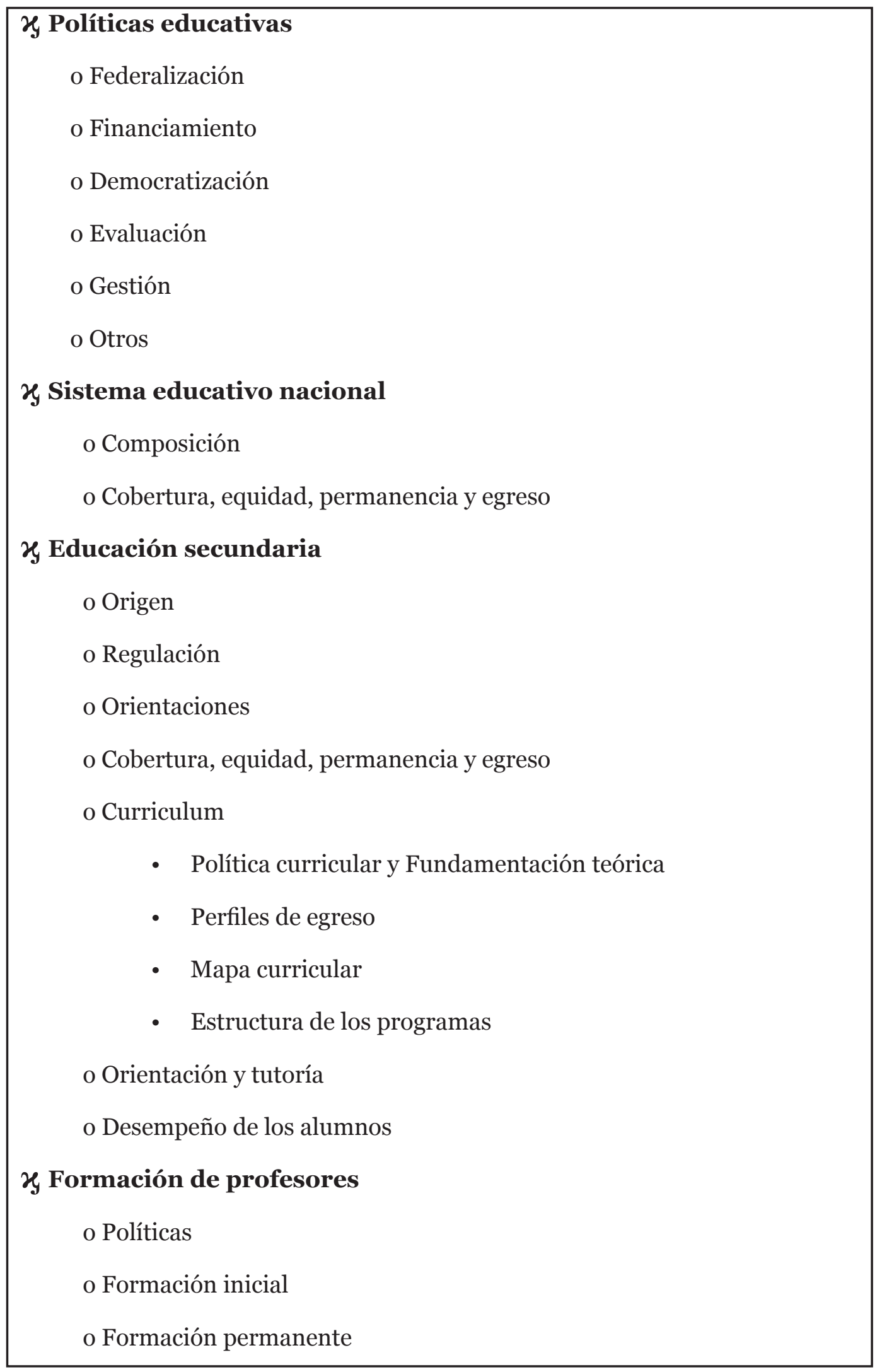

Fuente. Ducoing-Watty (2012). 
En tercer lugar proponemos desarrollar un ejercicio en paralelo, esto es, mediante la utilización de ambos dispositivos avanzar en la formulación de preguntas articuladas con las variables y los indicadores. Esta tarea permite orientar el trabajo de búsqueda, recopilación y sistematización de paquetes de datos para cada uno de los niveles y de cada una de las unidades de referencia (tratados, acuerdos, modelos, sistemas, sociedades, regiones y/o países) que han adquirido una preeminencia, o bien, han configurado un prototipo modélico reconocido en el panorama internacional, así como de las unidades de comparación (países, sistemas, comunidades y/o instituciones, culturas, grupos étnicos y otros semejantes) y sus respectivas variables.

En cuarto y último lugar, retomamos lo expuesto por Rojas-Moreno (2016a) en el sentido de enfatizar algunas de las acciones estratégicas clave para aplicar una metodología comparada como son las siguientes: a) seleccionar categorías de análisis pertinentes para demarcar redes de interrelación intra e interniveles; b) aplicar formulaciones teóricas sobre aspectos de un objeto de estudio, tal y como podrían ser «acuerdo», «modelo», «tratado, "programa», "pacto», «tendencia», etcétera, con base en las cuales se desarrollen descripciones analíticas y explicaciones argumentadas; c) realizar ejercicios de contraste entre unidades de comparatividad (estudios de caso de países, sistemas educativos, formas de gobernanza en comunidades y/o instituciones participantes); d) esbozar algunas vinculaciones hipotéticas entre los aspectos destacados de una determinada categoría (p. ej., si se trata de una determinada política educativa, situar sus alcances, impacto y efectos), enfatizando su articulación entre los diferentes niveles (internacional, regional, nacional, local) y los contextos socioculturales respectivos (país, cultura); e) esbozar un entretejido de este conjunto de articulaciones empíricas y las argumentaciones hipotéticas que presupone la investigación en curso, a fin de poner a prueba la capacidad de generalización teórica y de explicación sobre el qué, el cómo, el por qué y el para qué, por ejemplo, de una determinada agenda de política educativa.

\section{Estructura y avances de investigación comparada sobre educación secundaria: los casos de Francia y Brasil}

Previo a la presentación de resultados, destacamos algunos aspectos específicos sobre la estructura y la contextualización de nuestro protocolo de investigación del Proyecto de referencia (Cf. Ducoing-Watty, 2012).

\subsection{Etapas de investigación}

El trabajo realizado se desarrolló a lo largo de cuatro etapas. En la primera etapa utilizamos como estrategia básica los estudio de caso múltiples para aproximarnos al análisis del ciclo de educación secundaria en cada uno de los países que conformaron el universo de estudio (Cf. Nota 1), tomando como guía el desagregado del Esquema 1. El conjunto de procedimientos realizados para integrar paquetes de datos incluyó investigación documental, visitas a las escuelas secundarias de los países elegidos, entrevistas a funcionarios y personal docente, sistematización y análisis de fuentes bibliohemerográficas, textos digitalizados y referencias electrónicas, principalmente. En la segunda etapa considerada propiamente de integración de una visión panorámica de comparatividad 
multinivel, se delimitaron los ejes de estructura a partir de las siguientes preguntas: a) ¿Cómo se caracteriza la educación secundaria en el panorama internacional?; b) De acuerdo con las particularidades sociohistóricas y económicas de cada país, ¿cuáles son las semejanzas y diferencias de este nivel educativo?; y, c) ¿Cuál ha sido la influencia de los contextos nacionales en la configuración del currículum del nivel educativo en cuestión? En la tercera y última etapa más propiamente de análisis comparativo se avanzó en una demarcación panorámica sobre el nivel de educación secundaria en el contexto de la perspectiva internacional.

\subsection{Ejes y categorías análisis}

En cuanto a la definición de ejes transversales para la delimitación de un marco de referencia ad hoc y de acuerdo con el Esquema 1, utilizamos las categorías analíticas de «sistemas educativos», "política educativa sobre educación secundaria básica» y «currículum de educación secundaria», sobre las cuales mencionamos aquí breves puntualizaciones. En lo que se refiere a «sistemas educativos», ubicamos una referencia común utilizada por los expertos de la educación comparada: la consolidación de los Estados nacionales más desarrollados del continente europeo desde la Era Moderna como momento de anclaje para estudiar su surgimiento y conformación en diferentes países del mundo (Pedró, 1987). ${ }^{3}$

Respecto de la «política educativa sobre educación secundaria básica», en el marco del escenario internacional situamos el derecho a la educación reconocido en el Artículo 24 de la Declaración Universal de Derechos Humanos (UNESCO, 2008), el cual ha pasado a formar parte de una temática emergente y en paralelo como una demanda exigida por diversos organismos e instancias internacionales. ${ }^{4}$ Consideramos que en este entretejido se inscribe uno de los puntos de origen de la decisión global y de la definición de designar la educación secundaria como un elemento medular de las agendas de políticas educativas por parte de la comunidad internacional. Así, a lo largo de casi tres décadas el conjunto de Estados participantes ha combinado el refrendo de compromisos y esfuerzos políticos a fin de procurar la satisfacción de las necesidades de aprendizaje de niños y jóvenes a más tardar hacia finales de la presente década, de cara al parcial logro de dicho objetivo por parte de muchos otros países del orbe, principalmente los que aún no han podido alcanzar metas como esta. Es por ello que la consolidación de una agenda de política educativa de esta índole ha asentado la atención al sector poblacional de los adolescentes, quienes por encontrarse aún en edades de formación están expuestos a riesgos y amenazas como marginalidad social, explotación laboral, drogadicción, violencia, migración forzada e ilegal, embarazo prematuro y enfermedades como VIH SIDA, condiciones que limitan más todavía el aprovechamiento oportunidades de aprendizaje, constituyendo así un reto para los sistemas educativos. Recientemente y frente al mandato de ampliar la escolaridad obligatoria, la mayoría de los países ha puesto en marcha diversas estrategias diversas en este sentido en el marco de las respectivas agendas de políticas nacionales, revelándose entonces como un nivel educativo tendente a

3 Algunos antecedentes de interés pueden rastrearse en la obra de Dilthey de hace poco más de dos siglos, pasando por el estudio de los procesos fundacionales de instituciones educativas de diferentes niveles y grados, hasta situar en la actualidad los procesos de configuración de la mayoría de los sistemas educativos, de todos sus niveles, grados y modalidades.

4 Citamos aquí la Conferencia Mundial de Educación para Todos (1990) y el Marco de Acción de Dakar (2000) eventos ambos auspiciados por la UNESCO, el Programa de las Naciones Unidas para el Desarrollo (PNUD), el Fondo para la Infancia de las Naciones Unidas (UNICEF) y el Banco Mundial. 
universalizarse, en la perspectiva de coadyuvar al compromiso compartido por la comunidad internacional de ofrecer una educación básica para todos los ciudadanos y todas las sociedades.

Sobre la categoría de «currículum de educación secundaria», retomamos los aportes de Adamson y Morris (2010) en cuanto a las siguientes acepciones de currículum: a) herencia clásica a manera de asignaturas tradicionales; b) conocimiento organizado en ramas, ciencias o disciplinas que dan lugar al establecimiento de instituciones; c) utilidad social y económica de conocimientos y habilidades adquiridos; d) planificación de los resultados de aprendizaje; y, e) transformaciones personales, tanto de alumnos como docentes. A partir de estos referentes situamos la participación de entidades e instancias oficiales y no gubernamentales en la construcción nacional contextualizada de un modelo educativo impulsado desde planteamientos internacionales y universales. Lo anterior nos reafirma que en el marco de la educación comparada, el contraste entre modelos curriculares nacionales e internacionales es una tendencia que ha cobrado relevancia en las últimas décadas, no obstante la complejidad y diversidad de aspectos que todo currículum implica. En este sentido, compartimos lo expresado por los autores antes citados, en cuanto a enfatizar que el análisis comparativo de currículos es una modalidad de investigación de entidades «complejas y dinámicas» que se transforman continuamente.

\subsection{Contextualización de la investigación}

La denominación genérica de «educación secundaria» alude a la preparación de niños y jóvenes comprendida formalmente entre los 11 y 14 años de edad. Además de la delimitación convencional basada en el criterio de «edad ideal» para este nivel educativo, es importante situar la educación secundaria en relación con sus fines y alcances en contextos específicos y con contenidos curriculares determinados. Según Delors (1997), la educación secundaria constituye el nivel educativo por excelencia para consolidar las expectativas de formación de los sistemas educativos; siguiendo a este autor, al resaltar el carácter vestibular de este conjunto de estudios conviene no perder de vista que se trata de un período apropiado para detectar e impulsar en las poblaciones juveniles los talentos más variados.

Para efectos del presente artículo, la selección de los casos de Francia y Brasil de entre un universo de estudio más amplio (17 casos) está basada en proponer un comparativo sobre la educación secundaria en dos países representativos de contextos regionales contrastantes, como son la Unión Europea y la región Latinoamericana, además de considerar el desarrollo histórico diferenciado de sus respectivos sistemas educativos (Cf. Ducoing-Watty, 2017; Rojas-Moreno, 2016b). Algunos de los datos estadísticos que dan cuenta de esta situación se presentan en los cuadros siguientes. Por ejemplo, observamos diferencias sustanciales entre estos países a partir de rubros como el de Producto Interno Bruto (PIB) según el cual Brasil se encuentra aún por debajo del promedio de 25,908 dólares fijado por la Organización para la Cooperación y el Desarrollo Económico (OCDE), colocándose así en el lugar 101 del ranking mundial mientras que Francia está situada en el lugar 38. En cuanto al porcentaje de población ubicada por debajo del umbral de pobreza, en el caso de Brasil al menos una quinta parte de la población total se encuentra en una situación económica desventajosa (Ver Cuadro 1). 
Cuadro 1. PIB, índice de pobreza y sectores de la producción económica.

Fuente. Cuadro elaborado por las autoras con datos de Central Intelligence Agency (2017): The world factbook. Brazil, France (https://www.cia.gov/library/publications/the-worldfactbook/geos/br.html) consultado el 1o de marzo de 2017.

\begin{tabular}{|l|c|c|c|}
\hline PAÍS & $\begin{array}{c}\text { PIB PER CÁPITA } \\
\text { (DÓLARES } \\
\text { CORRIENTES) }\end{array}$ & $\begin{array}{c}\text { LUGAR EN EL } \\
\text { MUNDO }\end{array}$ & $\begin{array}{c}\text { POBLACIÓN CON } \\
\text { INGRESOS POR DEBAJO } \\
\text { DEL UMBRAL DE } \\
\text { POBREZA }\end{array}$ \\
\hline Francia & 42,400 & 38 & $8.1 \%$ \\
\hline Brasil & 15,200 & 101 & $21.4 \%$ \\
\hline
\end{tabular}

Otros datos de interés se destacan en la relación entre el número total de población, la población comprendida en el rango de edad de o a 14 años y de ese mismo rango la población escolarizada. En este rubro observamos que en ambos países la proporción de este grupo de edad ocupa casi una quinta parte del total de la población (18.59 \% en Francia frente a un $\mathbf{2 2 . 7 9} \%$ en Brasil). En cuanto a la escolarización para dicho rango el contraste es notable, puesto que en Francia poco más del 90 \% de ese grupo poblacional forma parte del sistema educativo, mientras que en Brasil la población escolarizada en ese mismo rango apenas rebasa el 30 \% (Ver Cuadro 2).

Cuadro 2. Datos sobre población total población menor de 15 años y población escolarizada. Fuente. Cuadro elaborado por las autoras con datos de Central Intelligence Agency (2017): The world factbook. Brazil, France (https://www.cia.gov/library/publications/the-worldfactbook/geos/br.html) consultado el 10 de marzo de 2017.

\begin{tabular}{|l|c|c|c|}
\hline \multicolumn{1}{|c|}{ PAÍS } & $\begin{array}{c}\text { POBLACIÓN } \\
\text { TOTAL }\end{array}$ & $\begin{array}{c}\text { POBLACIÓN DE O A 14 } \\
\text { AÑ OS }\end{array}$ & $\begin{array}{c}\text { POBLACIÓN DE O a } \\
\text { 14 ESCOLARIZADA }\end{array}$ \\
\hline Francia & $66,836,154$ & $12,425,212(18.59 \%)$ & $11,685,297(91.66 \%)$ \\
\hline Brasil & $205,823,665$ & $46,899,407(22.79 \%)$ & $15,944,063(32.6 \%)$ \\
\hline
\end{tabular}

\subsection{Caracterización de la educación secundaria}

En este apartado destacamos algunos de los hallazgos sobre los casos estudiados a modo de caracterizar los rasgos básicos de la educación secundaria en Francia y Brasil, comenzando por la estructura de los sistemas educativos. Cabe señalar que si bien en cada país la configuración del respectivo sistema educativo tiene sus propias especificidades históricas, político-administrativas e institucionales, en lo general ambos casos comparten elementos comunes en cuanto a la distribución de ciclos, niveles, grados y grupos de edad (Ver Cuadro 3). Ahora bien, tomando como criterio la demarcación etaria de la edad ideal (11 - 14 años) encontramos una denominación diferenciada que remarca, o bien el avance respecto de un nivel elemental (Francia: «Segundo nivel El Liceo»), o también la conclusión de un ciclo básico (Brasil: Educación final Años finales). En ambos casos la duración es similar (4 años) y el nivel educativo representa el vínculo con los subsiguientes niveles educativos. 
Cuadro 3. Estructura y organización de los sistemas educativos nacionales.

Fuente. elaboración de las autoras.

\begin{tabular}{|c|c|c|}
\hline NIVEL & $\begin{array}{c}\text { FRANCIA } \\
\text { (RANGO DE EDAD) }\end{array}$ & $\begin{array}{c}\text { BRASIL } \\
\text { (RANGO DE EDAD) }\end{array}$ \\
\hline $\begin{array}{l}\text { PREESCOLAR } \\
\text { (O EQUIVALENTE) }\end{array}$ & $\begin{array}{l}\text { Educación Pre-elemental / } \quad \text { Escuela } \\
\text { Materna o Preescolar (Duración: } 4 \text { años; } \\
\text { edad: } 2-6 \text { años) }\end{array}$ & $\begin{array}{l}\text { Educación maternal (Duración: } 4 \text { años; edad: } 0 \text { - } \\
3 \text { años) } \\
\text { Educación preescolar (Duración: } 2 \text { año; edad: } 4 \\
\text { - } 6 \text { años) }\end{array}$ \\
\hline $\begin{array}{l}\text { PRIMARIA } \\
\text { (O EQUIVALENTE) }\end{array}$ & $\begin{array}{l}\text { Primer Nivel / La Escuela (Duración: } 5 \\
\text { años; edad: } 6 \text { - } 11 \text { años) } \\
\text { Carácter: obligatorio }\end{array}$ & $\begin{array}{l}\text { Educación fundamental Ciclo inicial (Duración: } 5 \\
\text { años; edad: } 6 \text {-10 años) } \\
\text { Carácter obligatorio }\end{array}$ \\
\hline $\begin{array}{l}\text { SECUNDARIA } \\
\text { (O EQUIVALENTE) }\end{array}$ & $\begin{array}{l}\text { Segundo Nivel / El Colegio } \\
\text { (Duración: } 4 \text { años; edad: } 11-14 \text { años) } \\
\text { Carácter: obligatorio }\end{array}$ & $\begin{array}{l}\text { Educación fundamental Ciclo final } \\
\text { (Duración: } 4 \text { años; edad: } 11-14 \text { años) } \\
\text { Carácter: obligatorio }\end{array}$ \\
\hline $\begin{array}{l}\text { MEDIA SUPERIOR } \\
\text { (O EQUIVALENTE) }\end{array}$ & $\begin{array}{l}\text { Segundo Nivel / El Liceo } \\
\text { (Duración: } 3 \text { años; edad: } 15 \text { - } 17 \text { años) } \\
\text { Carácter: } 1 \text { año obligatorio }\end{array}$ & $\begin{array}{l}\text { Educación Secundaria Superior (Duración: } 3 \\
\text { años; edad: } 15-17 \text { años) }\end{array}$ \\
\hline $\begin{array}{l}\text { SUPERIOR } \\
\text { (O EQUIVALENTE) }\end{array}$ & $\begin{array}{l}\text { Examen de Bachillerato / Tercer Nivel } \\
\text { / Establecimientos (Universidades, } \\
\text { Institutos Universitarios de Tecnología, } \\
\text { Grandes Escuelas, escuelas dependientes } \\
\text { de otros Ministerios, Escuelas privadas }\end{array}$ & $\begin{array}{l}\text { Secuencias (Cursos secuenciales y } \\
\text { complementarios) } \\
\text { Graduación (Bachillerato, Licenciatura, } \\
\text { Tecnológico) } \\
\text { Posgrado (Maestría, Doctorado, Especialización, } \\
\text { Certificado de Especialización) }\end{array}$ \\
\hline
\end{tabular}

En lo que concierne a la obligatoriedad de la educación secundaria, encontramos que cada país ha establecido el cumplimiento de este rubro mediante la implantación de criterios específicos, concediendo el reconocimiento de su importancia al propiciar su integración al bloque de formación básica en los marcos de políticas nacionales y de tendencias internacionales de institucionalización de la educación (cobertura amplia, equidad, calidad, competencias, ciudadanía global, diversificación de servicios y modalidades, y evaluación). En el caso de Francia la escolarización obligatoria comprende diez grados grados (cinco de La Escuela, cuatro de El Colegio y uno de El Liceo), mientras que en el caso de Brasil la obligatoriedad abarca nueve grados del Nivel Fundamental (Años iniciales y Años finales) (Ver Cuadro 3).

Ahora bien, en el contraste sobre aspectos como formas de gobierno e implementación de políticas educativas resaltamos las siguientes diferencias. Como miembro de la Unión Europea, Francia define y orienta la agenda educativa nacional apegándose a los tratados, acuerdos y convenios signados en dicho marco, proclives al crecimiento socioeconómico de los países, basado en el aumento de la productividad y de la mano de obra altamente calificada, y en esa misma dirección se busca la articulación del sistema educativo. Bajo esta lógica, la definición y puesta en marcha de la política educativa está a cargo del gobierno francés, siendo en este caso el ministro de la educación nacional - asistido por el secretariado general- el responsable de su cumplimiento.

En el caso de Brasil, el Estado, a través del ejercicio gubernamental del Ministerio de Educación y Cultura y de los subsistemas que componen el sistema educativo (federal, estadual y municipal), ha trazado un plan para promover la enseñanza de calidad, 
iniciando a partir de la segunda mitad de la década pasada con la puesta en marcha de planes de desarrollo eucativo, en el sentido de construir una organización sistémica de los servicios educativos mediante estrategias de acción integradas a los planes y políticas nacionales para la obtención de espacios y financiamientos. En sus alcances están incluidas la educación básica lo mismo que la educación profesional y la educación superior, perfilándose hacia la implantación de una agenda educativa acorde en esencia con las directrices de organismos internacionales (Banco Mundial, Banco Interamericano de Desarrollo, CEPAL, UNESCO, OREALC). Todo este conjunto de acciones se ha encaminado al afianzamiento de las políticas neoliberales internacionales orientadas a la recentralización de los servicios, la ampliación de la oferta privada, el énfasis en la calidad y la evaluación de servicios y resultados.

Respecto de las estructuras de política y administración educativas, Francia se ha mostrado como uno de los países que, asumiendo la cada vez mayor interdependencia del mundo y junto con otros países de la región, impulsa un modelo de economía nacional basado en la mejora de los servicios educativos y de la formación, y presentado en términos de una política prioritaria promotora de la equidad y la cohesión social. Así, la organización político-administrativa nacional está articulada con la administración de la educación; para tal propósito en cada región y en cada departamento se han establecido servicios desconcentrados de la administración nacional. Este esquema de organización ha permitido mantener una gestión educativa coherente con el principio de servicio público del Estado, garantizando la supervisión de las instituciones y la implantación de reformas educativas acordes con los requerimientos de los contextos territoriales y comprometiendo la participación de los corresponsables locales.

En el caso de Brasil y acorde con la estructura del sistema educativo ya referida, para la configuración del currículum se cuenta, por una parte, con una organización políticoadministrativa derivada de una política de centralización respecto de la normatividad y la oficialización de los estudios. Por otra parte y considerando el supuesto de ampliación de cobertura de servicios, las provincias, estados y municipios se han atribuido facultades para definir de manera autónoma, pero con apego a las disposiciones federales sobre el currículum vigente, la oferta educativa tanto pública como privada. De hecho, desde la década de los noventa se inició una etapa de reforma curricular para la educación en todos sus niveles y modalidades, vinculada con las estrategias propuestas en la Conferencia Mundial de Educación Para Todos (Jomtiem, Tailandia, 1990). Fueron años de cambios orientados hacia el logro de metas como la reducción de las altas tasas de analfabetismo y la universalización de la enseñanza básica. Al inicio del nuevo milenio, el gobierno brasileño estableció un marco legal y filosófico para consolidar un esquema de educación nacional, definiendo principios y fines educativos además de las líneas básicas para la incorporación de las nuevas orientaciones curriculares para impulsar la reforma en educación básica (preescolar y fundamental). En lo que se refiere a la educación secundaria o Ciclo final de Educación fundamental, se ha propuesto el logro de las siguientes finalidades: a) preparación básica para el trabajo; b) formación ética y cívica; c) desarrollo de la autonomía intelectual y del pensamiento crítico; y, d) comprensión de los fundamentos científicos y tecnológicos. Cabe señalar también que en el caso de la educación básica y dado el carácter obligatorio de esta etapa formativa, los contenidos curriculares comparten una base nacional común.

Por último, en el marco de las consideraciones anteriores ubicamos ambas propuestas de formación académica tomando en cuenta el conjunto de asignaturas impartidas y la orientación formativa que sustenta cada uno de los currículos perfilados (Ver Cuadro 5). 
Cuadro 4. Formación académica en educación secundaria.

Fuente. elaboración de las autoras.

\begin{tabular}{|c|c|c|}
\hline PAÍS & ASIGNATURAS IMPARTIDAS & RASGOS DE ORIENTACIÓN FORMATIVA \\
\hline Francia & $\begin{array}{l}\text { Francés } \\
\text { Lengua extranjera: inglés } \\
\text { Lengua extranjera o regional } \\
\text { Matemáticas } \\
\text { Historia y geografía } \\
\text { Ciencias de la vida y la tierra } \\
\text { Tecnología } \\
\text { Ciencias físicas y química } \\
\text { Artes plásticas } \\
\text { Música } \\
\text { Educación física y deportiva }\end{array}$ & $\begin{array}{l}\text { Modelo de educación inclusiva para combatir el } \\
\text { racismo y la discriminación, promotora de la ciu- } \\
\text { dadanía, la aceptación de las diferencias de opi- } \\
\text { nión, de creencias y modos de vida. } \\
\text { Énfasis en la dimensión formativa de la educación } \\
\text { al fomentar los valores de la democracia y los de- } \\
\text { rechos fundamentales de la inclusión. } \\
\text { Fomento del espíritu crítico y el juicio, particular- } \\
\text { mente en el uso de las redes sociales, a fin de que } \\
\text { los adolescentes y jóvenes puedan distinguir la re- } \\
\text { alidad de las opiniones, de la propaganda, de los } \\
\text { discursos de odio, violencia e intolerancia. } \\
\text { Impulso del diálogo intercultural entre los actores } \\
\text { de la educación }\end{array}$ \\
\hline Brasil & $\begin{array}{l}\text { Lengua portuguesa } \\
\text { Matemática } \\
\text { Ciencias naturales } \\
\text { Historia } \\
\text { Geografía } \\
\text { Arte } \\
\text { Educación física } \\
\text { Lengua extranjera (inglés o español) } \\
\text { Temas transversales: Ética, Salud, Medio } \\
\text { ambiente, Orientación sexual, Pluralidad } \\
\text { cultural, Trabajo y consumo }\end{array}$ & $\begin{array}{l}\text { Modelo educativo centrado en los resultados del } \\
\text { aprendizaje, esto es, en estándares básicos de } \\
\text { competencias representativos de los referentes co- } \\
\text { munes que precisan los niveles de calidad a los que } \\
\text { tienen derecho todos los niños, niñas y jóvenes. } \\
\text { Logro de las siguientes finalidades: a) preparación } \\
\text { básica para el trabajo; b) formación ética y cívica; } \\
\text { c) desarrollo de la autonomía intelectual y del pen- } \\
\text { samiento crítico; y, d) comprensión de los funda- } \\
\text { mentos científicos y tecnológicos. }\end{array}$ \\
\hline
\end{tabular}

Cabe señalar que comparación entre Francia y Brasil deja entrever cosmovisiones abismales entre el continente europeo y la región latinoamericana. Por una parte ubicamos el referente del debate actual que, al menos en Europa, se sostiene sobre la pertinencia de diversificar la educación secundaria diferenciando la formación propedéutica destinada a los estudiantes talentosos, de la preparación de carácter tecnológico y/o terminal ofertada a los estudiantes menos favorecidos. Por otra parte, en la región latinoamericana se ha obviado esta discusión concentrando el esfuerzo de los gobiernos en consolidar la oferta de este nivel educativo en dos vertientes paralelas: la educación secundaria como el ciclo propedéutico para la educación superior, o bien, la del ciclo que complementa y cierra la etapa infantil, brindando una preparación incipiente para la incorporación de los jóvenes al mercado de trabajo.

En breve, atendiendo a los elementos de contraste de los casos presentados, reafirmamos el siguiente argumento: los modelos curriculares de educación secundaria sean de corte academicista o de orientación pragmática, al igual que el conjunto de los sistemas 
educativos, son resultado de tendencias y procesos políticos, sociales, económicos y educativos de largo aliento. No obstante, llama la atención el hecho de que la educación secundaria se ha universalizado en un lapso comprendido entre la última década del siglo XX el momento actual en los casos de países con un desarrollo económico más retrasado o aquellos considerados como economías emergentes.

\section{Discusión y conclusiones}

En este último apartado nos enfocamos en resaltar aquellas preguntas que nos han guiado para preparar el contenido del presente artículo, como son las siguientes: ¿cómo realizar investigación educativa basada en una aproximación de corte comparativo?, ¿qué pautas teórico-metodológicas pueden ayudar a definir un esquema de investigación?, ¿qué tipo de resultados pueden obtenerse?, ¿qué utilidad ofrece esta manera de investigar?, ¿̇cuáles son sus alcances y sus limitaciones? Aunque al momento de este cierre no pretendemos responder en forma definitorio las cuestiones anteriores, sí queremos presentarlas como hilos conductores de los que se deriven algunas reflexiones que nos permitan apreciar avances y perspectivas en el ámbito de la educación comparada.

Por ejemplo, una revisión selectiva y detallada de la amplia producción sobre el tema nos ha permitido detectar ciertas similitudes en el desarrollo de estrategias de investigación comparada. Así, encontramos que los estudios de caso incluidos en compilaciones articuladas a partir de un núcleo o de eje temático transversal representan el ejemplo recurrente a la vez que sugestivo, para resolver en parte el problema del «cómo hacer» de esta estrategia de aproximación hacia un determinado objeto de estudio. Asimismo, estimamos que los referentes metodológicos rastreados en otras investigaciones representan apenas una parte del trabajo de rastreo de orientaciones metodológicas que no siempre son aplicables a cualquier tipo de investigación comparada. En ese sentido es que nos interesa enfatizar que la definición de coordenadas de investigación tiene que entrelazarse con una visión de «abordar lo complejo» partiendo de acciones básicas de organización y definición de estructuras de trabajo. Recursos como el ejercicio de comparación multinivel de casos, la búsqueda de redes de interrelación, la demarcación de contextos sociohistóricos, entre otros apoyos que hemos destacado en este artículo, ofrecen múltiples posibilidades para detectar hallazgos observando la variedad de posibilidades de un objeto de investigación como las aristas de un poliedro.

Ejercicios como la descripción, el contraste, los desagregados, la multiplicidad de conexiones posibles, el acceso de una búsqueda a profundidad y los alcances de una lectura de superficie, representan todo un conjunto de acciones o equipaje para el acompañamiento que brinda la construcción metodológica para proporcionar el «andamiaje» de la investigación y apoyar su posterior desarrollo. Así, más que hablar de la utilidad de la comparatividad resaltamos la riqueza de este tipo de trabajos de búsqueda y de producción de conocimientos.

Finalmente, enfatizar la aplicación de estos dispositivos a manera de herramientas de soporte no se opone a estimar hasta dónde puede llegar una investigación, sus alcances y limitaciones para comprender y caracterizar procesos tales como tendencias convergentes, implementación de políticas educativas, implantación de modelos curriculares, formas de gestión y gobernanza nacionales y/o locales, , entre otros aspectos temáticos susceptibles de convertirse en objetos de estudio, y en los cuales la educación comparada pueden ayudarnos a vislumbrar la complejidad implícita, así como a integrar un panorama de contraste mediante el cual sea factible detectar problemas comunes y definir estrategias de atención validadas en contextos diferentes. 


\section{Fuentes de consulta}

ADAMSON, B. \& P. MORRIS (2010): La comparación de currículos, en M. BRAY et al. (Eds.), Educación comparada. Enfoques y métodos, pp. 321-342 (Buenos Aires, Granica).

ALTBACH, Ph. \& KELLY, G. (1990): Nuevos enfoques en educación comparada (Madrid, Mondadori).

BEECH, J. \& MEO, A. I. (2016): Explorando el uso de las herramientas teóricas de Stephen J. Ball en el estudio de las políticas educativas en América Latina, Revista Archivos analíticos de políticas públicas. Vol. $24 \mathrm{~N}^{\circ}$ 23, pp. 1-19. (http://www. redalyc.org/articulo.oa?id=275043450048), consultado el 27 de marzo de 2017.

CHANE-DAVID, F. \& GROUX, D. (Coords.) (2009): Méthodologie de la comparaison en éducation (París, L'Harmattan).

CENTRAL INTELLIGENCE AGENCY (2017): The world factbook Brazil, France, European Union (https://www.cia.gov/library/publications/the-world-factbook/ geos/br.html) consultado el 10 de marzo de 2017.

DELORS, J. (1997): La educación encierra un tesoro. Informe a la UNESCO de la Comisión Internacional sobre la Educación para el siglo XXI (París: UNESCO).

DUCOING-WATTY, P. (2012): Proyecto PAPIIT Clave IN 402712 La Educación Secundaria: retos y perspectivas al inicio del siglo. Estudio comparado (México, UNAM/Documento institucional).

DUCOING-WATTY, P. (2017): 13. Francia: Un acercamiento al Colegio Único, en Ducoing-Watty, La Educación Secundaria en el mundo: el mundo de la Educación Secundaria Vol. IV, pp. 277-413 (México, UNAM/En prensa).

KIN, R. (1994): Case study research. Design and methods (London, Sage Publications).

PEDRÓ, F. (1987): Los precursores españoles de la educación comparada (Madrid, Ministerio de Educación y Ciencia).

ROJAS-MORENO, I. (2016a): Educación comparada: apuntes y reflexiones sobre la configuración y la delimitación de un ámbito del conocimiento educativo, Revista Academicus, 1-8, pp. 17-29 (http://www.ice.uabjo.mx/media/15/2017/05/ Art8_2.pdf), consultado el 23 de abril de 2017.

ROJAS-MORENO, I. (2016b): 9. La educación secundaria en Brasil, en Ducoing-Watty, La Educación Secundaria en el mundo: el mundo de la Educación Secundaria Vol. III, pp. 16-82 (México, UNAM/En prensa).

SCHRIEWER, J. (1993): El método comparativo y la necesidad de externalización: criterios metodológicos y conceptos sociológicos, en J. SCHRIEWER \& F. PEDRÓ (Eds.), Manual de educación comparada (Vol. II) Teorías, investigaciones, perspectivas, pp. 189-251 (Barcelona, Pomares-Corredor). 
SCHRIEWER, J. (2011): Sistema mundial y redes de interrelación: la internacionalización de la educación y el papel de la investigación comparada, en CARUSO, M., \& TENORTH, H-E. (Comps.), Internacionalización. Políticas educativas y reflexión pedagógica en un medio global, pp. 41-105 (Buenos Aires, Granica).

STAKE, R. (1999): Investigación con estudio de casos (Madrid, Morata).

STAINER-KHAMSI, G. (2015): 2. La transferencia de políticas como herramienta para comprender la lógica de los sistemas educativos, en RUIZ, G. \& ACOSTA, F. (Eds.), Repensando la educación comparada: lecturas desde Iberoamérica: Entre los viajeros del siglo XIX y la globalización, pp. 55-74 (Barcelona, Ediciones Octaedro).

UNESCO-OREALC (2008): Declaración Universal de Derechos Humanos, Santiago de Chile (http://unesdoc.unesco.org/images/o017/o01790/179018m.pdf), consultado el 6 de abril de 2016.

VALLE, J. M. et al. (2016): Investigación en Educación Comparada: Pistas para investigadores noveles, Revista Latinoamericana de Educación Comparada, 7 - 1, pp. 39-56 (http://www.saece.org.ar/relec/revistas/9/revista_a7n9.pdf\#page=39) consultado el 18 de mayo de 2017.

WALLERSTEIN, I. (2013): Análisis de sistemas-mundo. Una introducción (México: Siglo XXI). 